\title{
THE DETERMINATION OF SOME PROPERTIES OF A FUNCTION SATISFYING A PARTIAL DIFFERENTIAL EQUATION FROM ITS SERIES DEVELOPMENT
}

\author{
STEFAN BERGMAN
}

1. The method of integral operators. The solution of an equation and its associate. The method of integral operators in the theory of linear partial differential equations of the type ${ }^{1}$

$$
\begin{aligned}
L(U) & \equiv\left(U_{x x}+U_{y y}\right) / 4+A(x, y) U_{x} / 2+B(x, y) U_{y} / 2+C(x, y) U \\
& \equiv U_{z \bar{z}}+2 \operatorname{Re}\left[a(z, \bar{z}) U_{z}\right]+c(z, \bar{z}) U=0
\end{aligned}
$$

where

$$
\begin{gathered}
z=x+i y, \quad \bar{z}=x-i y, \quad U_{z}=[(\partial U / \partial x)-i(\partial U / \partial y)] / 2, \\
U_{\bar{z}}=[(\partial U / \partial x)+i(\partial U / \partial y)] / 2,
\end{gathered}
$$

consists in associating with an arbitrary analytic function $f(\zeta)$ of a complex variable $\zeta$, by means of an operator of the form

$$
\begin{aligned}
& U(z, \bar{z})=M(f) \equiv \operatorname{Re}[P(f)] \\
& u(z, \bar{z})=P(f)=\int_{-1}^{1} \mathbf{E}(z, \bar{z}, t) f\left(z\left(1-t^{2}\right) / 2\right) d t /\left(1-t^{2}\right)^{1 / 2}
\end{aligned}
$$

a solution $U(z, \bar{z})$ of the equation (1.1).

$\mathbf{E}=\mathbf{E}(z, \bar{z}, t),|t| \leqq 1$, is any analytic function of $z$ and $\bar{z}$ which satisfies the equation

$$
G(\mathrm{E}) \equiv\left(1-t^{2}\right)\left(\mathrm{E}_{\bar{z} t}+a \mathrm{E}_{t}\right)-t^{-1}\left(\mathrm{E}_{z}+a \mathrm{E}\right)+2 z t L(\mathrm{E})=0,
$$

is regular in a sufficiently large domain and has the property that $\left(\mathrm{E}_{\bar{z}}+A \mathrm{E}\right) / z t$ is continuous at $\bar{z}=0, t=0$.

REMARK. An operator (1.2) is determined by choosing a particular function $\mathbf{E}$ (the generating function of the operator) which satisfies the above requirements.

Let $U(z, \bar{z})$ be a function which satisfies the equation $L(U)=0$ and which is an entire function of two complex variables $x$ and $y$, that is, a solution of (1.1) whose series development

\footnotetext{
Presented to the Society, April 28, 1944; received by the editors December 10, 1943.

${ }^{1}$ Since we consider the functions $u(z, \bar{z})$ for real values of $x$ and $y$, that is for $z$ and $\bar{z}$ conjugate, it would be, of course, sufficient to write simple $u(z)$. We shall, however, use the first notation in order to stress the fact that $u(z, \bar{z})$ is a (complex) analytic function of two real variables $x, y$, reserving $u(z)$ for analytic functions of one complex variable.
} 


$$
U(z, \bar{z})=\sum_{m, n=0}^{\infty} B_{m n} x^{m} y^{n} \equiv \sum_{m, n=0}^{\infty} D_{m n} z^{m} \bar{z}^{n}, \quad D_{m n}=\bar{D}_{n m},
$$

converges for $|x|<\infty,|y|<\infty$.

The problem with which we shall be concerned in $\$ \S 2$ and 3 of this note is to determine an upper bound for the growth of $U(x, y)$ for real values of $x, y$ from certain subsequences of the coefficients, $D_{m n}$, of its series development (1.5). Further we shall give in $\$ 4$ a condition in terms of $A_{m 0}$ for $V=\sum_{m, n=0}^{\infty} A_{m, n} z^{m} \bar{z}^{n}, L(V)=0$, to have only one branch point of certain type on its circle of regularity.

If $f(z / 2)$ is an analytic function which is regular in the circle $|z| \leqq \rho$, then clearly $u(z, \bar{z})$ will be an analytic function of two real variables in the circle $x^{2}+y^{2}<\rho^{2}$. The converse is not necessarily true.

If $\mathrm{E}(z, \bar{z}, t)$ possesses the property that to every $u(z, \bar{z})$ which is regular in a circle $x^{2}+y^{2}<\rho^{2}$ there corresponds a unique function $f(z / 2)$ which is regular in the circle $|z|<\rho_{1}, \rho_{1}>0$ and independent of $f$, then we shall say that $\mathrm{E}$ is a generating function of the type $\mathcal{A}$, or that $\mathrm{E}$ belongs to $\mathcal{A}$. If in addition to this, for every $f, \rho_{1}=\rho$ then $\mathrm{E}$ will be said to be a generating function belonging to $B$. In a previous paper, [1a], ${ }^{2}$ it was shown that to every equation $L(U)=0$ where $a$ and $c$ are entire functions of $z$ and $\bar{z}$, there exists an $\mathrm{E}(z, \bar{z}, t)$ (a generating function of the first kind) of the form

$$
\mathrm{E}(z, \bar{z}, t)=\left[\exp \left(-\int_{0}^{\bar{z}} a(z, \bar{z}) d \bar{z}\right)\right]\left[1+z \bar{z} t^{2} \mathrm{E}^{*}(z, \bar{z}, t)\right]
$$

where $\mathrm{E}^{*}$ is a regular function.

The operator (1.3) where $\mathbf{E}$ is of the form (1.6) possesses the property that if $u(z, \bar{z})$ is regular in a simply connected domain which includes the origin, then $f(z / 2)$ is also regular in this domain, and hence $\mathbf{E}$ in this case belongs to $\mathcal{B}$.

The function $f$ which upon application of the operator $P$ yields $u$ will be denoted as the associate of $u$ with respect to the generating function $\mathbf{E}$. (Often, however, we omit the last phrase.)

REMARK. If we change the generating function $\mathrm{E}$, we change the law connecting the real and imaginary parts, $U$ and $V$, of the ob-

2 The numbers in brackets refer to the following papers: 1. S. Bergman, a. Rec. Math. (Mat. Sbornik) N.S. vol. 2 (1937) pp. 1169-1198; b. Trans. Amer. Math. Soc. vol. 53 (1943) pp. 139-155; c. The hodograph method in the theory of compressible fluids, Brown University, 1942; d. Proc. Nat. Acad. Sci. U.S.A. vol. 29 (1943) pp. 276-281; 2. L. Bers and A. Gelbart, Quarterly of Applied Mathematics vol. 1 (1943) pp. 168188. 3. L. Bieberbach, Funktionentheorie, vol. 2, 1927; 4. G. Darboux, La theorie generale de surfaces, vol. 2, 1915; 5. K. L. Nielsen and B. P. Ramsay, Bull. Amer. Math. Soc. vol. 49 (1943) pp. 156-162; 6. A. Zygmund, Trigonometrical series, 1935. 
tained (complex) solution, $u$. That is to say, if for a given $L$ we have two generating functions $\mathrm{E}_{1}$ and $\mathrm{E}_{2}$ and two functions $f_{1}, f_{2}$, such that $U_{1}+i V_{1}=\boldsymbol{P}_{1}\left(f_{1}\right)$ and $U_{1}+i V_{2}=\boldsymbol{P}_{2}\left(f_{2}\right)$, then $V_{1}$ in general differs from $V_{2}$.

In the case of the generating function of the first kind the corresponding complex solutions

$$
u(z, \bar{z})=\sum_{m, n=0}^{\infty} A_{m n} z^{m \bar{z}^{n}}, A_{00} \text { real, }
$$

have the property that

$$
\begin{aligned}
& c_{m} A_{m 0}=a_{m}, \quad c_{m}=2^{m} \Gamma(m+1) / \pi^{1 / 2} \Gamma(m+1 / 2), \\
& \sum_{m=0}^{\infty} A_{0 m} \bar{z}^{m}=A_{00} \exp \left[-\int_{0}^{z} a(0, \bar{z}) d \bar{z}\right],
\end{aligned}
$$

where the $a_{m}$ are the coefficients of the series development $f(\zeta)$ $=\sum_{n=0}^{\infty} a_{n} \zeta^{n}$ of the associate function $f$.

In this case we may also express the $a_{m}$ in terms of the coefficients $D_{m n}$ of the real part of $u(z, \bar{z}), U(z, \bar{z})$. Substituting $\bar{z}=0$ in

$$
\begin{aligned}
\sum_{m, n=0}^{\infty} D_{m n} z^{m_{\bar{z}}^{n}}= & \frac{1}{2} \int_{-1}^{1}\left[\mathrm{E}(z, \bar{z}, t) f\left(z\left(1-t^{2}\right) / 2\right)\right. \\
& \left.+\overline{\mathrm{E}}(\bar{z}, z, t) \bar{f}\left(\bar{z}\left(1-t^{2}\right) / 2\right)\right] d t /\left(1-t^{2}\right)^{1 / 2}
\end{aligned}
$$

we have by (1.5)

$$
\begin{aligned}
\sum_{m=0}^{\infty} D_{m 0} z^{m}=\frac{1}{2} & \left\{\sum_{m=0}^{\infty} 2^{-m} a_{m} \Gamma(m+1 / 2) \pi^{1 / 2}\right)[\Gamma(m+1)]^{-1} z^{m} \\
& \left.+\bar{f}(0) \pi \exp \left(-\int_{0}^{z} \bar{a}(0, z) d z\right)\right\}
\end{aligned}
$$

Thus from given $D_{m 0}$ and $\bar{a}(0, z)$ we may determine the $a_{m}$ and, therefore, by the classical results of the theory of functions the order of the growth of the associate function $f$ in (1.3). Consequently if in addition we know the order of the growth of $\mathbf{E}$ we can obtain by (1.3) an upper bound for the order of the complex solution, and hence for its real part, $U$.

2. Relations between the series developments of $U$ and its associate in the general case. However in applying this method it is extremely important to obtain the lowest possible order of growth for the function $\mathbf{E}$, and it is for this reason that in some instances we shall consider functions $\mathrm{E}$ belonging to $\mathcal{A}$ but of different structure 
than those of the first kind. The price we have to pay for this is that the laws connecting the coefficients $D_{m n}$ and the $a_{m}$ will be considerably more complicated than those which follow from (1.8).$^{3}$ If now we put $\bar{z}=0$ in (1.9) and set

$$
\frac{1}{2} \int_{-1}^{1} \overline{\mathrm{E}}(0, z, t) d t /\left(1-t^{2}\right)^{1 / 2}=\sum_{m=0}^{\infty} L_{m} z^{m}
$$

then we obtain

$$
\sum_{\nu=0}^{n} a_{\nu} P_{n, \nu}=2 D_{n 0}-2 \bar{f}(0) L_{n}
$$

where

$$
\begin{aligned}
P_{n, \nu} & =2^{\nu} \int_{-1}^{1} P_{n}(t)\left(1-t^{2}\right)^{\nu-1 / 2} d t, \quad \nu \geqq n ; \quad P_{0, \nu} \neq 0, \\
\mathrm{E}(z, 0, t) & =\sum_{\nu=0}^{\infty} P_{\nu}(t) z^{\nu} .
\end{aligned}
$$

Conversely, we have

(2.4) $\quad a_{n}=\left|\begin{array}{lllll}P_{0,0} & 0 & 0 & \cdots 2\left(D_{00}-\bar{f}(0) L_{0}\right) \\ P_{1,0} & P_{0,1} & 0 & \cdots 2\left(D_{10}-\bar{f}(0) L_{1}\right) \\ \cdot & \cdot & \cdot & \cdots & . \\ P_{n, 0} & P_{n-1,1} & P_{n-2,2} & \cdots & 2\left(D_{n 0}-\bar{f}(0) L_{n}\right)\end{array}\right| / \prod_{\nu=0}^{n} P_{0, \nu}$ instead of (1.8).

3. Upper bounds for the growth of generating functions $\mathbf{E}$ of equations $L$, whose coefficients are connected by certain differential relations. In $\S 3$ of [1] a procedure was indicated to determine the differential equations $L$, a generating function of which is of the form

$$
\mathrm{E}(z, \bar{z}, t)=\exp [\mathrm{P}(z, \bar{z}, t)]
$$

where $\mathbf{P}$ is a polynomial in $t$.

Nielsen and Ramsay in [5] applied this method and found additional equations $L$ with generating functions (3.1). In the latter pa-

${ }^{8}$ When we use rough estimates for the growth of $\mathbf{E}(z, \bar{z}, t)$, for example like those given on p. 1176 of [1a], then the results are valid for a very large class of equations $L$. The majorant for $\mathrm{E}$ derived in Theorem 2 of [1a] depends only upon the maxima of $|a|$ and $|c|$ in certain domains. The estimates which we obtain in the following use the property of the coefficients $a$ and $c$ in a much more specific fashion. The estimates are more special and more precise. (Note that the $P_{n, \nu}$ depend upon $\mathbf{E}$, and therefore upon $a$ and $c$.) 
per, however, the conditions are formulated in a form which is not very convenient for immediate application. The equation (1.1) is assumed already to be reduced to the form $u_{z}+D u_{z}+F u=0$, and the conditions are given in the form of assumptions concerning the structure of $D$ and $F$. It seems useful for applications to formulate the same conditions in the form of differential relations between the coefficients as was done in Theorem 4 of [1a ].4

As we shall see, in certain cases several types of the generating functions (3.1) are admissible.

CAsE 1.

$$
a_{z}=\bar{a}_{\bar{z}}, \quad F_{z}=0, \quad F \equiv-a_{z}-|a|^{2}+c .
$$

Then

$$
\mathrm{P}=-\int_{0}^{\bar{z}} a d \bar{z}+z^{1 / 2}\left[C-4 \int_{0}^{\bar{z}} F d \bar{z}\right]^{1 / 2} t
$$

or

$$
\mathrm{P}=-\int_{0}^{\bar{z}} a d \bar{z}-k_{1} z+z^{1 / 2}\left[-4 \int_{0}^{\bar{z}} F d \bar{z}\right]^{1 / 2} t+k_{1} z t^{2} .
$$

Case 2.

$$
a_{z}=\bar{a}_{\bar{z}}, \quad \phi=2\left(z F_{\bar{z}}-F\right) / F_{z} F_{z \bar{z}}=\text { constant. }
$$

Then

$$
\mathrm{P}=-\int_{0}^{z} a d \bar{z}-z^{1 / 2} \phi^{-1 / 2}\left(z+\phi F_{z}\right) t+2 \phi^{-1 / 2} t^{3} / 3 .
$$

Case 3.

$$
F_{z}=0, \quad F=\left(a_{z}-\bar{a}_{\bar{z}}\right) / 2
$$

Then

$$
\mathrm{P}=-\int_{0}^{\bar{z}} a d \bar{z}+z\left[C+2 \int_{0}^{\bar{z}} F d \bar{z}\right] t^{2}
$$

where $C$ is an arbitrary constant.

Case 4.

$$
\bar{a}_{\bar{z}}-a_{z}=F, \quad F_{z}=0 .
$$

"We note that line 4 from bottom, p. 1197 of [1a ], should be " $z^{8 / 2} g M_{\bar{z}}=0$ " instead of " $z g^{8 / 2} M_{z}=0$ " and line 2, p. 1197, " $h_{z}=-A$ " instead of " $h_{\bar{z}}=-A$." 
Then

$$
\begin{aligned}
\mathrm{P}= & -\int_{0}^{z} \bar{a} d z+\int_{0}^{z} \int_{0}^{z} F d z d \bar{z}+C z \\
& -\left[2 C z^{2}+z \int_{0}^{z} F d \bar{z}\right] t^{2}+C z^{2} t^{4}
\end{aligned}
$$

where $C$ is an arbitrary constant.

The procedure indicated in [1a] and [5] yields further types of equations $L$ which possess a generating function of the form (3.1).

The method described in $\S 3$ of [1a] can be immediately generalized for a much larger class of equations $L$.

Consider the equation $u_{x y}+a(x, y) u_{x}+b(x, y) u_{y}+c(x, y) u=0$ whose coefficients are supposed to be (complex) analytic functions of two real variables, and where $b(x, y)=\bar{a}(y, x)$. If we replace $x$ by $z$ and $y$ by $\bar{z}$ then the latter equation becomes $L(u)=0$ (see (1.1)). Generalizing the "method of cascade" to the case of the equations $L(u)=0$, we form the invariants ${ }^{5}$

$$
\begin{aligned}
& F^{(0)}, F^{(1)}, \cdots, \\
& F^{(0)}=-F \equiv a_{z}+|a|^{2}-c, \\
& F^{(n+1)}=2 F^{(n)}-\bar{F}^{(n)}-F^{(n)} \Delta F^{(n)} / 4+\left|F_{z}^{(n)}\right|^{2},
\end{aligned}
$$

and the corresponding equations

$$
\begin{aligned}
& L_{0}, L_{1}, L_{2}, \cdots, \\
& L_{0}=L, L_{n}(v)=v_{z z}+a^{(n)} v_{z}+b^{(n)} v_{z}+c_{v}^{(n)}
\end{aligned}
$$

where

$$
\begin{aligned}
& a^{(n+1)}=a^{(n)} F_{z}^{(n)} / F^{(n)}, \quad b^{(n+1)}=b^{(n)}, \\
& c^{(n+1)}=c^{(n)}-a_{z}^{(n)}-b_{z}^{(n)}-2 b^{(n)} F_{z}^{(n)} / F^{(n)}, \quad b^{(0)}=\bar{a} .
\end{aligned}
$$

See $[4$, p. 28$]$.

If the coefficients of one of the equations $L_{n}$ satisfy any one of the conditions (3.4), (3.5), (3.7), (3.9) or similar relations then the gen-

8 If an invariant, say $F^{(n)}$, vanishes identically and, therefore, the sequence (3.11) consists of only a finite number of nonvanishing terms, then the solutions of $L(u)=0$ may be represented in the form $u(z, \bar{z})=\sum_{k=0}^{n} B_{k}(z, \bar{z}) d^{k} f(z) / d z^{k}$, where the $B_{k}$ are certain fixed functions, and $f(z)$ ranges over the field of analytic functions of a complex variable $z$. See, for example, $[4$, p. 42] or $[1 \mathrm{a}$, Theorem 4$]$. 
erating function of $L_{n}$ has the form (3.1). Hence the expression

$$
\begin{aligned}
u(z, \bar{z}) & =N\left[\int_{-1}^{1} e^{\mathrm{P}(z, \bar{z}, t)} f\left(\bar{z}\left(1-t^{2}\right) / 2\right) d t /\left(1-t^{2}\right)\right] \\
& =\int_{-1}^{1} N\left[e^{\mathrm{P}(z, \bar{z}, t)}\right] f\left(\bar{z}\left(1-t^{2}\right) / 2\right) d t /\left(1-t^{2}\right)^{1 / 2},
\end{aligned}
$$

where $\boldsymbol{N}$ denotes the differential operator

$$
\begin{array}{r}
N_{[m]} \equiv\left[\exp \left(-\int_{0}^{z} \tilde{a} d z\right)\right] \frac{1}{F^{(0)}} \frac{\partial}{\partial z}\left\{\frac { 1 } { F ^ { ( 1 ) } } \frac { \partial } { \partial z } \left[\frac{1}{F^{(2)}}\right.\right. \\
\left.\left.\cdots\left(\frac{1}{F^{(n-1)}} \frac{\partial}{\partial z}\left(m \exp \left(\int \bar{a} d z\right)\right)\right)\right]\right\}
\end{array}
$$

and $f(\zeta)$ is an arbitrary analytic function, will be a solution of $L(u)=0$. See $[4$, p. 30]. Therefore

$$
\mathrm{E}(z, \bar{z}, t)=N\left[e^{\mathrm{P}(z, \bar{z}, t)}\right]
$$

is a generating function of $L(u)=0$.

EXAMPLE. If $L_{1}$ satisfies the conditions (3.2), then

$$
N(m)=\left[\exp \left(-\int_{0}^{z} \bar{a} d z\right)\right] \frac{\partial}{\partial z}\left[m \exp \left(\int_{0}^{z} \bar{a} d z\right)\right] / F^{(0)} .
$$

Substituting $m=\left[z\left(C-4 \int_{0}^{z} F^{(1)} d \bar{z}\right)\right]^{1 / 2} t$ yields

$$
\begin{aligned}
\mathrm{E}=\left\{\left(b-a^{(1)}\right) F^{-1}+\right. & \frac{t}{2}\left[\left(C-4 \int_{0}^{z} F^{(1)} d \bar{z} / z\right]^{1 / 2} F^{-1}\right\} \\
\cdot & \exp \left\{-\int_{0}^{\bar{z}} a^{(1)} d \bar{z}+\left[z\left(C-4 \int_{0}^{z} F^{(1)} d \bar{z}\right)\right]^{1 / 2} t\right\} .
\end{aligned}
$$

If we now assume that the growth of the coefficients $a$ and $c$ of $L$ is known, then using the above results we can easily determine an upper bound for the growth of E. For instance, if $a$ and $c$ are polynomials, then we obtain an upper bound for $\mathbf{E}$ of the form

$$
\exp \left(A|z|^{n}\right) \text {. }
$$

Now, two cases can occur. Either the order of growth of $f$ is more than $n$, then the growth of $f$ is decisive for the growth of $u(z, \bar{z}),{ }^{6}$ or the growth of $f$ is less than $n$, in which case a rough estimate of the

6 The order of growth of $f$ can be determined from the coefficients $A_{m 0}$ (or $D_{m 0}$ ) of series developments of (1.7) or (1.5). 
growth of the right-hand side of (1.3) yields the order $n$. In many instances, however, we may obtain much sharper bounds. It may happen that the order of growth of $\exp [\mathrm{P}(z, \bar{z}, t)]$ in the real $x y$ plane or at least for some values of $\phi=\arg z$ is smaller than $n$. Then it is sometimes possible to find estimates for the order of growth of $u(z, \bar{z})$ from the $A_{m 0}$ or $D_{m 0}$. In such cases we may obtain from $A_{m 0}$ an upper bound for the growth of $u$ in the whole plane or at least in some directions, despite the fact that the order of growth of $f$ is less than $n$.

Finally we may determine solutions of (1.4) which are bounded at infinity if we approach along a certain direction. We can then obtain upper bounds for the growth of $u$ from the $A_{m 0}$ in this direction.

EXAMPLE. Let $L(u) \equiv \Delta u+u=0$. Then $\mathbf{E}(z, \bar{z}, t)=\cos \left[t(z \bar{z})^{1 / 2}\right]$ is bounded in the whole plane, and we obtain immediately an upper bound in terms of the $a_{m}$ (and therefore in terms of the $A_{m 0}$ or of the $\left.D_{m 0}\right)$ for

$$
u(z, \bar{z})=\int_{-1}^{1} \cos \left[t(z \bar{z})^{1 / 2}\right]\left\{\sum_{n=0}^{\infty} 2^{-n} a_{n} z^{n}\left(1-t^{2}\right)^{n-1 / 2}\right\} d t .
$$

In this connection it would also be possible to apply the well known results on the growth of the function $f$ in a specific direction from the coefficients of its series development.

REMARK. In previous papers [1c, p. 23], [1d, p. 277] (see also [2]) an operator, $T$, was introduced. $T$ is of a different form than (1.3). It transforms the power series, $s=\sum_{n=0}^{\infty}\left(\alpha_{n}+i \beta_{n}\right)(x+i Y)^{n}$, into functions $\sum_{n=0}^{\infty}\left(\alpha_{n}+i \beta_{n}\right)(x+i Y)^{[n]}$ whose imaginary parts satisfy the equation

$$
l(Y) U_{x x}+U_{Y Y}=0 .
$$

Concerning the definition of $(x+i Y)^{[n]}$, see (2.3) of [1d] and p. 23 of [1c]. After the introduction of a suitable variable, $y=y(Y)$, the equation (3.17) assumes the form

$$
L(U)=U_{x x}+U_{y y}+N(2 y) U_{y}=0 .
$$

Let $T^{*}$ be the operator which we obtain from $T$ by replacing $Y$ by $Y(y)$. If the equation (1.1), or one of the equations $L_{n}$ of the sequence (3.12) corresponding to $L$, has the form (3.19) then by applying the operator $N T^{*}$ to a power series, $s$, we obtain a solution of (1.1).

The same reasoning can be applied to all types of equations considered by Bers and Gelbart in [2].

4. Conditions for a branch point on the boundary of the largest regularity circle. As was proved previously the radius, $\rho$, of the largest 
circle with the center at the origin in which $u(z, \bar{z})=\sum_{m, n=0}^{\infty} A_{m n} z^{m} \bar{z}^{n}$ is regular is given $b^{7}$

$$
\rho=1 / \limsup _{n \rightarrow \infty}\left|A_{n 0}\right|^{1 / n} .
$$

A problem which now arises is to determine how many singular points lie on the boundary of the circle, and the character of these singularities. There exists a very simple test (the test of Hadamard) in the case of analytic functions to decide that on the largest regularity circle one and only one pole lies.

The theory of operators enables us to obtain a similar procedure to decide that one and only one singular point of a certain type lies on the largest circle of regularity. In order to make the essential idea of the procedure clearer we shall at first apply it in the case where $u(z, \bar{z})=u(z)$ is an analytic function. Let $Q^{(n)}(z), n=0,1,2, \cdots$, be a set of analytic functions of a complex variable $z$, such that

$$
\sum_{n=0}^{\infty}\left|z^{n} Q^{(n)}(|z|)\right|
$$

converges uniformly in a sufficiently large circle, say for $|z| \leqq \rho^{*}$.

The point $2 a$ is said to be a branch point of the type $\left\{Q^{(n)}\right\}$ of $u$ if the function $u$ can be represented in the neighborhood of $2 a$ in the form

$$
\begin{aligned}
u(z)=\pi^{1 / 2} \sum_{n=0}^{\infty} 2^{-2 n} \Gamma(2 n+1) & {[\Gamma(n+1)]^{-1} Q^{(n)}(z) } \\
\cdot & I^{1 / 2+n}\left[z^{-1 / 2}(a-z / 2)^{-1}\right]+g(z)
\end{aligned}
$$

where $g(z)$ is a regular function at the point $z=2 a$, and the $I^{k}$ denote the integral of $k$ th order. (For the definition of the integrals of nonintegral order see [6, p. 222].)

REMARK. We have

$$
\begin{aligned}
& \begin{aligned}
I^{1 / 2}\left[z^{-1 / 2}(a-z / 2)^{-1}\right] & =\pi^{-1 / 2} \int_{-1}^{1}\left[a-z\left(1-t^{2}\right) / 2\right]^{-1}\left(1-t^{2}\right)^{-1 / 2} d t \\
& =\pi^{1 / 2} a^{-1 / 2}(a-z / 2)^{-1 / 2}, \\
& =2 \pi^{-1 / 2} \int_{-1}^{1 / 2} t^{2}\left[a-z\left(1-z^{-1 / 2}(a-z / 2]^{-1}\left(1-t^{2}\right)^{-1 / 2} d t\right.\right. \\
& =2 \pi^{1 / 2} z^{-1}(a-z / 2)^{-1 / 2}\left[a^{1 / 2}-(a-z / 2)^{1 / 2}\right] .
\end{aligned}
\end{aligned}
$$

7 See [1b, p. 142, formula (7.4)]. We note that this formula should be "lim $\sup _{n \rightarrow \infty}\left(A_{n 0}\right)^{1 / n}$ " instead of " $\lim _{n \rightarrow \infty}\left(A_{n 0} c_{n}\right)^{1 / n}$." 
THEOREM. A. A sufficient condition that

$$
u(z)=\sum_{m=0}^{\infty} A_{m} z^{m}
$$

possess (as a unique singularity on the circle of convergence, $|z|=2|a|$ ) a branch point of the type $\left\{Q^{(n)}(z)\right\}$ at $z=2 a$ is that there exists a constant $k, k<1$, such that

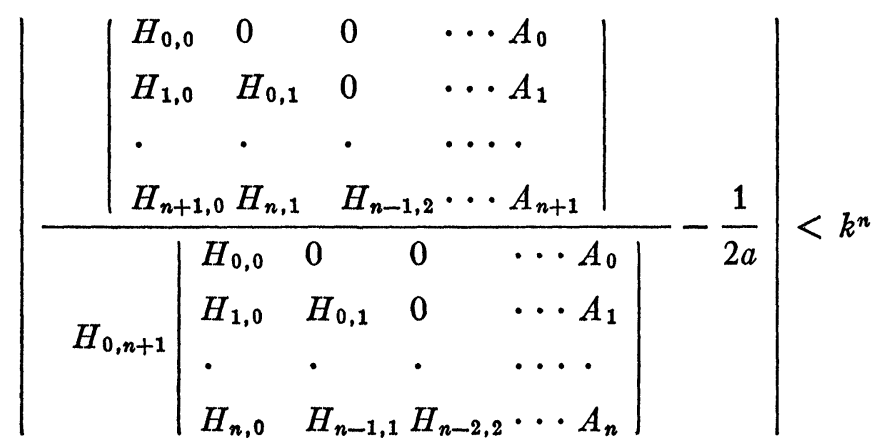

where

$$
H_{n, k}=\int_{-1}^{1} H_{n}(t)\left(1-t^{2}\right)^{k-1 / 2} d t / 2^{k}
$$

and the $H_{n}(t)$ are given by

$$
\mathrm{E}(z, t)=\sum_{n=0}^{\infty} t^{2 n} z^{n} Q^{(n)}(z)=\sum_{\nu=0}^{\infty} H_{\nu}(t) z^{\nu} .
$$

B. If $\mathrm{E}(z, t) \in \mathcal{B}$, see p. 559 , then the above condition is also necessary.

REMARK. We assume that the radius of convergence, $\rho^{*}$, of $(4.2)$ is larger than $\rho$ given by (4.1).

Proof. A. We note that

$$
\begin{aligned}
& \int_{-1}^{1} \mathrm{E}(z, t)\left[a-z\left(1-t^{2}\right) / 2\right]^{-1} d t /\left(1-t^{2}\right)^{1 / 2} \\
& \quad=\sum_{n=0}^{\infty} 2^{-2 n} \Gamma(2 n+1)[\Gamma(n+1)]^{-1} Q^{(n)}(z) I^{n+1 / 2}\left[z^{-1 / 2}(a-z / 2)^{-1}\right] .
\end{aligned}
$$

We consider now the function

$$
f(z)=\sum_{n=0}^{\infty} a_{n} z^{n}
$$


where

$$
a_{n}=\left|\begin{array}{lllll}
H_{0,0} & 0 & 0 & \cdots & A_{0} \\
H_{0,0} & H_{0,1} & 0 & \cdots & A_{1} \\
\cdot & \cdot & \cdot & \cdots \\
H_{n, 0} & H_{n-1,1} & H_{n-2,2} & \cdots & A_{n}
\end{array}\right| / \prod_{\nu=0}^{n} H_{0, \nu}
$$

By (4.6) the radius of convergence of this series is $2|a|$ and by the Hadamard test (see, for example, [3, p. 219]) the series is regular in the circle $|z| \leqq 2|a|$ except at the point $z=2 a$, where it possesses a simple pole. Therefore

$$
f(z / 2)=C_{0}(a-z / 2)^{-1}+f_{1}(z / 2)
$$

where $f_{1}(z)$ is regular for $|z| \leqq|a|$.

$$
\begin{aligned}
\int_{-1}^{1} \mathrm{E}(z, t) f\left(z\left(1-t^{2}\right) / 2\right) d t /\left(1-t^{2}\right)^{1 / 2} \\
=\int_{-1}^{1} \mathrm{E}(z, t) f_{1}\left(z\left(1-t^{2}\right) / 2\right) d t /\left(1-t^{2}\right)^{1 / 2} \\
\quad+C_{0} \int_{-1}^{1} \mathrm{E}(z, t)\left[a-z\left(1-t^{2}\right) / 2\right]^{-1} d t /\left(1-t^{2}\right)^{1 / 2}
\end{aligned}
$$

will be a function which is regular for $|z| \leqq 2|a|$ except at $z=2 a$, where it has a branch point of the type $\left\{Q^{(n)}\right\}$. See (4.9). On the other hand a formal computation yields

$$
u(z)=\sum_{n=0}^{\infty} A_{n} z^{n}=\sum_{n=0}^{\infty} z^{n}\left(\sum_{\nu=0}^{n} 2^{-v} a_{\nu} H_{n-\nu, \nu}\right)
$$

and therefore $f(z)=\sum_{n=0}^{\infty} a_{n} z^{n}$, with $a_{n}$ given by (4.11), is the associate of $u(z)$ with respect to $\mathbf{E}(z, t)$. Hence (4.6) is a sufficient condition.

B. Suppose now that $\mathbf{E}(z, t) \in \mathcal{B}$ and that $u(z)$ is regular in $|z| \leqq 2|a|$ except at the point $z=2 a$, where $u(z)$ possesses a branchpoint of the type $\left\{Q^{(n)}\right\}$. Hence

$$
\begin{aligned}
u_{1}(z)=u(z)-C_{0}\left[\sum_{n=0}^{\infty} 2^{-2 n} \Gamma(2 n+1)\right. & {[\Gamma(n+1)]^{-1} Q^{(n)}(z) } \\
\cdot & \left.I^{n+1 / 2}\left[z^{-1 / 2}(a-z / 2)^{-1}\right]\right]
\end{aligned}
$$

is regular in $|z| \leqq 2|a|$. Since $\mathbf{E} \in \mathbb{B}$, it follows that the associate $f_{1}(z)$ 
of $u_{1}(z)$ is regular for $|z| \leqq|a|$, and therefore denoting by $f$ the associate of $u$ we obtain

$$
f(z / 2)=f_{1}(z / 2)+C_{0}(a-z / 2)^{-1} .
$$

As we have previously shown, the coefficients $a_{n}$ of $f(z)$ are given by (4.11). Since the Hadamard relation is a necessary condition in order that $f(z)$ is regular in $|z| \leqq 2|a|$ except for a simple pole at $z=2 a$, it follows that in the case $\mathbf{E}(z, t) \in \mathcal{B},(4.6)$ is a necessary condition.

Clearly, using the results of Hadamard, the same procedure may be applied to obtain conditions that $u(z, \bar{z})$ possesses several branchpoints of the type $\left\{Q^{(n)}\right\}$.

The generalization of the theorem to the case where $u(z, \bar{z})$ is a solution of the equation (1.1) is evident. Now the functions $Q^{(n)}(z, \bar{z})$ are no longer arbitrary; for in addition to the fact that $\sum_{n=0}^{\infty}\left|z^{n} Q^{(n)}(|z|,|\bar{z}|)\right|$ converges uniformly,

$$
\sum_{n=0}^{\infty} t^{2 n} z^{n} Q^{(n)}(z, \bar{z})
$$

must be a solution of the equation (1.4).

If we denote by $A_{m n}$ the coefficients of the series development (1.7) of $u(z, \bar{z})$ and by the $D_{m n}$ the coefficients of the development (1.5) of the real part $U$ of $u$, then the $a_{n}$ can be determined from either the subsequence $A_{m 0}$ or from the $D_{m 0}$ 's. In the first case we must replace in (4.11) the $A_{m}$ by the $A_{m 0}$ and obtain the $H_{n, k}$ by substituting in (4.7) the coefficients $H_{\nu}(t)$ of the series development

$$
E(z, 0, t)=\sum_{k=0}^{\infty} t^{2 k} z^{k} Q^{(k)}(z, 0)=\sum_{\nu=0}^{\infty} H_{\nu}(t) z^{\nu}
$$

Similar results may be obtained if we express $a_{n}$ in terms of the $D_{m 0}$.

BROWN UNIVERSITY 